\title{
Equivalent Modelling of Reciprocating Engines Generators for Microgrid Frequency Response Analysis
}

\author{
F. Conte, S. Massucco, F. Silvestro \\ DITEN \\ Università degli Studi di Genova \\ Genova, Italy \\ \{fr.conte, stefano.massucco, federico.silvestro\}@unige.it
}

\author{
F. Baccino, P. Serra \\ ABB SPA Power Grids \\ Division BU Grid Automation \\ Genova, Italy \\ \{francesco.baccino, pietro.serra\}@it.abb.com
}

\begin{abstract}
Typical microgrid configurations include small-scale generation units that belong to the class of reciprocating engines (gas, diesel, heavy fuel oil). A simplified equivalent model for representing the frequency response of a given set of this kind of generators is proposed in this paper. The model parameters are tuned to obtain frequency responses compliant with the performance classes stated by the ISO 8528-5 standard. The result is a set of equivalent and simple models that can be used to simulate the frequency response within a given microgrid configuration that includes a set of reciprocating engines generators. Finally, a suitable validation of the proposed models is carried out using two highly detailed models of real diesel and heavy fuel oil generators.
\end{abstract}

Index Terms-Microgrids, power system simulation, frequency response, diesel engines, ISO standards.

\section{INTRODUCTION}

During last years, the industrial and academic research has focused to the development of several management solutions and applications in order to ensure reliable, secure, and economical operations of microgrids [1]-[4]. Before transferring the proposed solutions to real applications, an analysis of effectiveness is required. Simulation is a valid instrument to show the expected advantages and drawbacks of a given solution since it can be used to evaluate the expected results, but also to calibrate and tune the system parameters, such as the dimensions and the number of the involved units [5].

Many microgrid configurations include a certain number of small-scale power generation units such as diesel, bio-diesel [6], liquefied petroleum gas (LPG) [7], heavy fuel oil (HFO), or compressed natural gas (CNG) engines [8]. All of them belong to the class of reciprocating engines generators (REGs), which use one or more reciprocating pistons to convert pressure into a rotating motion. Because of the growing interest in high RES penetration driven by carbon and fuel saving needs, the dynamic behavior of this type of equipment is becoming more and more interesting. In particular, mainly from the industrial community, simplified analysis tools are required to allow not highly skilled personnel to reasonably size the different devices composing a given microgrid using a limited amount of information.
The main objective of this work is the development of a simple and fast simulation tool to analyze the frequency response of a set of different REGs in a microgrid configuration. A general single unit model is firstly considered based on the existing literature [9]-[12]. Then an equivalent single unit representation is proposed for a set of REGs within a given microgrid. The simplified model parameters are tuned in order to obtain a generator response compliant with the performance classes defined in the ISO 8528-5 standard [13]. The result is a library of simple models of "standard generators" which can be used to readily simulate the microgrid frequency dynamics, requiring a limited amount of information, such as number of generators, nominal powers and performance classes. Finally, two detailed and validated generator models are taken as benchmark to verify that the proposed modelling can actually be tuned to picture generators dynamics.

The rest of the paper is organized as it follows. The equivalent model is presented in Section II. Section III provides the tuned model parameters according to the ISO standard. Section IV describes the validation tests. Finally, conclusions are presented in Section V.

\section{The EQuivalent MODEL}

\section{A. Single unit model}

There are many methods proposed for modelling the frequency-power dynamics of REGs [9]-[12]. All of these use the linear approximation. The schematic structure assumed in this work is shown in the following Fig. 1, where the overline sign indicates a per unit (p.u.) quantity or transfer function and $\Delta x$ indicates the perturbation of the variable $x$ with respect to the current operating point.

In the figure: $\overline{\Delta \varepsilon}_{f}$ is the angular speed error, $\overline{\Delta \Omega}_{r e f}$ is the refer mechanical angular speed, $\overline{\Delta \Omega}_{m}$ is the current mechanical angular speed, $K_{2}$ and $\tau_{2}$ are the fuel actuator system gain and time constant, respectively, $K_{3}$ is the current driver gain, $\bar{\Phi}$ is the fuel flow, $\bar{T}_{m}$ is the mechanical torque, $\tau_{1}$ is the engine time delay, $K_{1}$ is the torque constant, $\overline{\Delta T}_{L}$ models the total disturbance due to power unbalances arising 


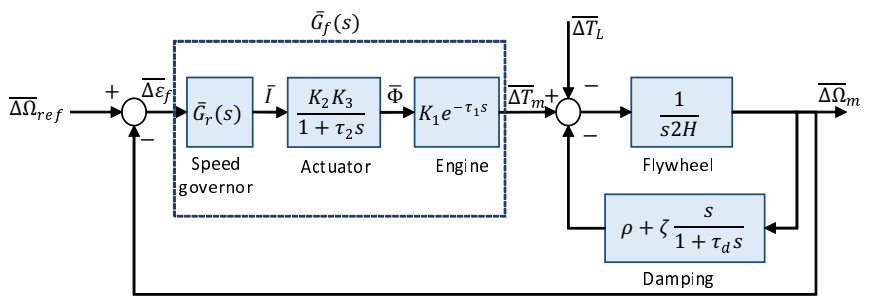

Fig. 1. Per unit block diagram of a typical REG.

in the network, $H$ is the flywheel inertia constant, and $\rho$ is the damping coefficient.

Moreover, notice that a further damping term, scaled by the coefficient $\zeta$, is added to the model. Thanks to its high-pass filter form, it contributes only to transient phases ( $\tau_{d}$ is present only for realizability and it must be set to values sufficiently close to zero, in order to be practically negligible). Such an additive term represents electromechanical transient damping phenomena, which are usually neglected by most of the literature models [9]-[12]. However, in some configurations for very fast generators, they should be considered to obtain a consistent modelling.

The values of $K_{3}$ and $K_{2}$ can be considered to be constant for a particular engine setup. Gain $K_{3}$ is a factor that determines the amount of the mechanical torque obtained per unit of fuel flow. It depends on the operating point of the prime mover. Time constant $\tau_{2}$ is quite dependent on the temperature of the oil flowing into the actuator. Both $K_{2}$ and $\tau_{2}$ are variable, but their variation is negligible in a small time interval.

The speed governor is supposed to be a standard proportional integral (PI) controller: $\bar{G}_{r}(s)=K_{P}+K_{I} / s$. Therefore, by defining $K_{0}=K_{1} K_{2} K_{3}$ and $\tau_{3}=K_{P} / K_{I}$, the p.u. transfer function $\bar{G}_{f}(s)$ assumes the form

$$
\bar{G}_{f}(s)= \begin{cases}\frac{K_{0} K_{I}\left(1+\tau_{3} s\right)}{s\left(1+\tau_{2} s\right)} e^{-\tau_{1} s}, & K_{I} \neq 0 \\ \frac{K_{0} K_{P}}{1+\tau_{2} s} e^{-\tau_{1} s}, & K_{I}=0\end{cases}
$$

depending on the presence or not of the integral action.

The answer to a variation of the power balance arising into the network can be described by the so-called power-frequency transfer function, which, in this particular case, assumes the following form (omitting the negligible damping term $1+\tau_{d} s$ ):

$$
\frac{\overline{\Delta \Omega}_{m}(s)}{\overline{\Delta P}_{L}(s)}=-\frac{1}{\bar{G}_{f}(s)+\rho+\zeta s+2 H s}
$$

where $\Delta P_{L}$ is the power load disturbance $\left(P_{L}=\Omega_{m} T_{L}\right)$. Denoting by $b_{p}$ the speed droop (ratio between steady state p.u. frequency variation and p.u. power variation), from (2) it follows that, with an integral action $\left(K_{I} \neq 0\right), b_{p}=0$, whereas, without integral action $\left(K_{I}=0\right), b_{p}=1 /\left(K_{0} K_{P}+\rho\right)$. Note that for standard values of droop lower than $10 \%$, considering that $\rho \simeq 0.1$ for REGs [9], [12], $b_{p}$ can be computed as $b_{p}=1 / K_{0} K_{P}$ with negligible errors.

\section{B. Sensitivity on time-delay}

Since small REGs are particularly fast, time-delays cannot be considered negligible. This is clear in Fig. 2, which reports the Bode diagram of the open loop transfer function of the model in Fig. 1 and the time response of the power-frequency transfer function (2) to a $-10 \%$ power load step, with and without considering the time-delay $\tau_{1}$. Note that at the cutoff frequency $\nu_{t}=1.25 \mathrm{rad} / \mathrm{s}$ the discrepancy due to the time-delay is significant. This is generally not true for larger generators, which usually have a lower cut-off frequency $\nu_{t} \sim$ $0.3-0.5 \mathrm{rad} / \mathrm{s}[14]$.
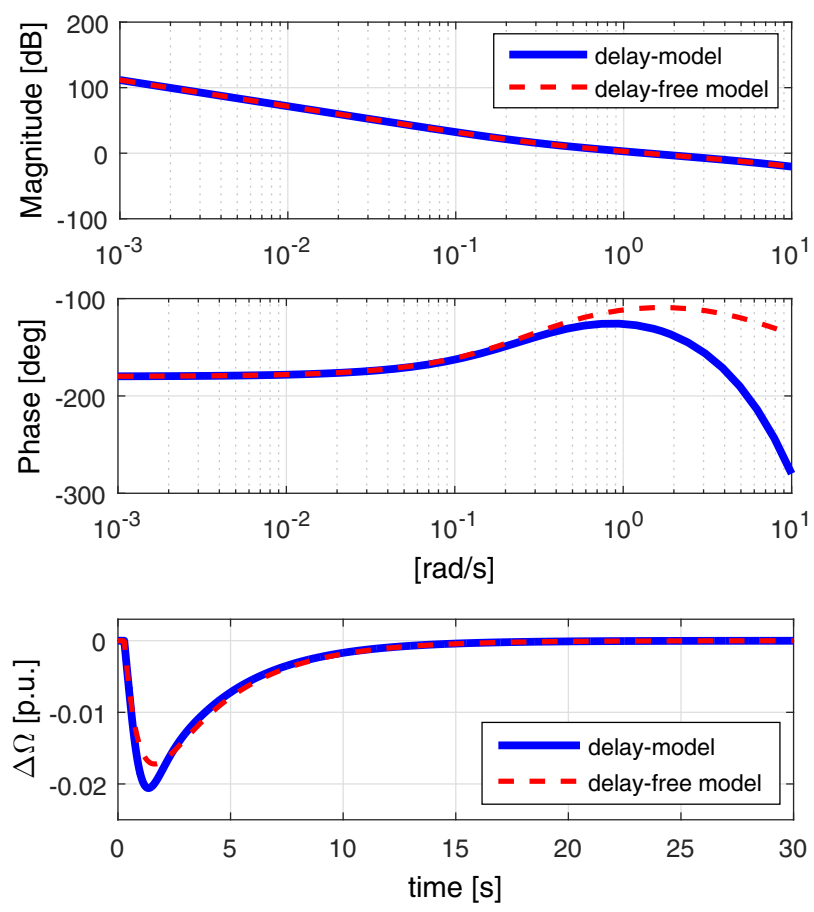

Fig. 2. Comparison of simulated models of a gas generator with (solid blue) and without (dashed red) time-delay $\tau_{1}=0.25 \mathrm{~s}$ : Bode diagram of the open loop transfer function of the model in Fig. 1 (top) and time response of the power-frequency transfer function (2) to a $-10 \%$ power load step (bottom).

\section{Multiple unit system model}

Consider now a multiple unit system (MUS) composed by $N$ synchronous generation units, all operating speed regulation and modelled as in Section II-A. Hereafter, the subscript $i$ will indicate a quantity referred to the $i$-th generation. Define $P_{\text {nom }, i}$ as the nominal power of the $i$-th generation unit, $P_{\text {nom }}=\sum_{i=1}^{N} P_{\text {nom }, i}$ as the nominal power of the overall MUS, and $\bar{P}_{\text {nom }, i}=P_{\text {nom }, i} / P_{\text {nom }}$ as the percentage nominal power of the $i$-th unit.

The main assumption required to simplify the analysis of such a system is that the electric speed is, even during transients, equal for all the units, as if they were, at the same number of pole pairs, mechanical coupled one the other. This hypothesis is generally acceptable in particular for small networks such as microgrids, where the transient slips between the machines vanish quite rapidly because of the synchronizing 
actions [14]. With this simplification, the modelling scheme becomes that pictured in Fig. 3, where $\Omega_{m}$ is the synchronous mechanical angular speed (for simplicity, all generators are supposed to have the same number of pole pairs) and $H, \rho$, and $\zeta$ are the MUS inertia constant, the friction coefficient, and the additive damping coefficient, respectively defined as $H=\sum_{i=1}^{N} \bar{P}_{\text {nom }, i} H_{i}, \rho=\sum_{i=1}^{N} \rho_{i}$, and $\zeta=\sum_{i=1}^{N} \zeta_{i}$.

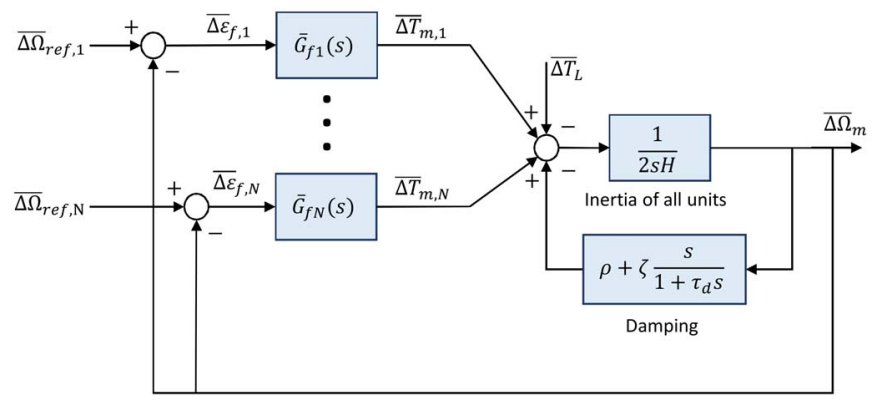

Fig. 3. Multiple unit system (MUS) block diagram.

Concerning the time constants $\tau_{d, i}$, recall that they must be set to be negligible. Therefore, they can be considered all equal to the same value $\tau_{d}$, which is used in the MUS.

The MUS can be represented by a high-order single unit model with the form in Fig. 1 with

$$
\bar{G}_{f}(s)=\sum_{i=1}^{N} \bar{P}_{\mathrm{nom}, i} \bar{G}_{f, i}(s) .
$$

The resulting model is extremely complex because in (3) there is the sum of first order transfer function with timedelays. Following an approach similar to the one used in [14] for large traditional generators, but taking to account the presence of the engines time-delays, which are negligible for large generators, an approximated equivalent single unit model can be obtained by replacing (3) with:

$$
\bar{G}_{f}^{m}(s)= \begin{cases}\frac{K^{m}\left(1+\tau_{3}^{m} s\right)}{s\left(1+\tau_{2}^{m} s\right)} e^{-\tau_{1}^{m} s}, & K_{I, i} \neq 0 \text { for any } i \\ \frac{K^{m}}{1+\tau_{2}^{m} s} e^{-\tau_{1}^{m} s}, & K_{I, i}=0 \text { for all } i\end{cases}
$$

where:

$$
\begin{gathered}
K^{m}=\sum_{i=1}^{N} K_{i}^{m}, \\
K_{i}^{m}=\left\{\begin{array}{cc}
\bar{P}_{\text {nom }, i} K_{0, i} K_{I, i}, & K_{I, i} \neq 0 \\
\bar{P}_{\text {nom }, i} K_{0, i} K_{P, i}, & K_{I, i}=0
\end{array},\right. \\
\tau_{2}^{m}=\frac{1}{K^{m}} \sum_{i=1}^{N} K_{i}^{m} \tau_{2, i}, \quad \tau_{3}^{m}=\frac{1}{K^{m}} \sum_{i=1}^{N} K_{i}^{m} \tau_{3, i}, \\
\tau_{1}^{m}=\frac{1}{K^{m}} \sum_{i=1}^{N} K_{i}^{m} \tau_{1, i} .
\end{gathered}
$$

Using the equivalent single unit model, the p.u. powerfrequency transfer function assumes the form

$$
\frac{\overline{\Delta \Omega}_{m}(s)}{\overline{\Delta P}_{L}(s)}=-\frac{1}{\bar{G}_{f}^{m}(s)+\rho+\zeta s+2 H s}
$$

and the equivalent droop $b_{p}$ is zero if one of the units has $K_{I, i} \neq 0$ or equal to $b_{p}=1 / K^{m}$ if no unit operates integral action.

Based on a suitable analysis, the proposed equivalent model is consistent if the following conditions are satisfied: a) the electric speed is, even during transients, equal for all the units; b) the time constants $\tau_{2, i}$ have the same order of magnitude; c) the time-delays $\tau_{1, i}$ are almost equal, namely $\left|\tau_{1, i}-\tau_{1, j}\right|<$ $0.15 \mathrm{~s}$ for all $i, j=1,2, \ldots, N$.

Condition a) is generally acceptable in particular for small networks, such as microgrids. Condition b) is a hypothesis required also in the case of large power generators as analyzed in [14]. Condition c) is due to the sensibility of small REGs to the time-delays discussed in Section II-B. The limit value of $0.15 \mathrm{~s}$ has been obtained through a simulation analysis, which compares the Bode diagrams of the openloop transfer function and the step responses of the powerfrequency transfer function computed using the complete highorder model and the equivalent single unit model. Note that a microgrid generally includes a set of REGs with similar size and technology. Therefore, conditions b) and c) can be considered to be satisfied for most of microgrids cases.

\section{Model TUning For ISO STANDARD CLASSIFICATION OF RECIPROCATING ENGINES}

The aim of this section is to build a library of simple models for standard REGs which can be used to simulate the frequency response within a given microgrid configuration that includes a set of REGs. This is done by tuning the proposed single unit equivalent model in order to make the generator response compliant with the ISO 8528-5 standard [13]. The ISO standard specifies the requirements for the frequency profiles, according to three different performance classes: G1, G2 and G3. The requirements are different for diesel and gas fuelled generators. The standard refers to the four parameters listed in Table I:

TABLE I

ISO 8528-5 GENERATOR PERFORMANCE SPECIFICATIONS.

\begin{tabular}{lcccc}
\hline \multicolumn{1}{c}{ Parameter } & \multicolumn{4}{c}{ Performance classes } \\
& G1 & G2 & G3 \\
\hline & & & & \\
Frequency droop [\%] & $\leq 8$ & $\leq 5$ & $\leq 3$ \\
Related frequency tolerance band [\%] & 3.5 & 2 & 2 \\
Frequency recovery time [s] & & $\leq 10$ & $\leq 5$ & $\leq 3$ \\
Maximum transient frequency & Diesel & -15 & -10 & -7 \\
Deviation from rated value [\%] & Gas & -25 & -20 & -15 \\
\hline
\end{tabular}

and defined as it follows. Frequency droop $b_{p}$ : the ISO standard states three maximum values of the frequency droop for each class; however, for some applications, the required frequency droop is $0 \%$ (isochronous). The related frequency tolerance band $\alpha_{f}$ defines the steady-state frequency tolerance band $\Delta f_{\text {tol }}$ by $\Delta f_{\text {tol }}=\alpha_{f} f_{\text {nom }} / 100$. The frequency recovery time is time interval between the departure from the steadystate frequency band after a sudden specified load increase or decrease and the permanent re-entry of the frequency into 
the specified steady-state frequency tolerance band $\Delta f_{\text {tol }}$. The maximum transient frequency deviation is the maximal variation of the frequency from the rated value after a $100 \%$ power load increase.

Table II lists the two sets of parameters tuned for each fuel type, each performance class, and three different machine sizes (different inertia time constants).

TABLE II

DIESEL AND GAS REGS STANDARD PARAMETERS SETS COMPLIANT WITH ISO 8528 PRESCRIPTIONS

\begin{tabular}{ccccccc}
\hline \multirow{2}{*}{ Class } & $H$ & $K_{I}$ & $K_{p}$ & $\tau_{1}[\mathrm{~s}]$ & $\zeta$ & $\tau_{2}[\mathrm{~s}]$ \\
& {$[\mathrm{s}]$} & {$[p . u] *$.} & {$[p . u] *$.} & & {$\left[\% P_{\text {nom }} / \mathrm{Hz}\right]$} & isoch \\
& & & & \\
\hline
\end{tabular}

\begin{tabular}{|c|c|c|c|c|c|c|c|}
\hline \multicolumn{8}{|c|}{ Diesel } \\
\hline $\mathrm{G} 1^{1}$ & 0.4 & 6 & 4 & 0.05 & 11 & 0.125 & 0.625 \\
\hline $\mathrm{G}^{2}{ }^{2}$ & 0.7 & 6 & 3 & 0.1 & 11 & 0.125 & 0.625 \\
\hline $\mathrm{G}^{3}{ }^{3}$ & 1.5 & 6 & 2 & 0.05 & 11 & 0.125 & 0.625 \\
\hline $\mathrm{G} 2^{1}$ & 0.4 & 18 & 6 & 0.02 & 13 & 0.125 & 0.375 \\
\hline $\mathrm{G}^{2}{ }^{2}$ & 0.7 & 18 & 6 & 0.08 & 13 & 0.125 & 0.375 \\
\hline $\mathrm{G} 2^{3}$ & 1.5 & 6 & 6 & 0.13 & 13 & 0.125 & 0.375 \\
\hline $\mathrm{G}^{1}{ }^{1}$ & 0.4 & 12 & 15 & 0.01 & 15 & 0.125 & 0.250 \\
\hline $\mathrm{G}^{2}{ }^{2}$ & 0.7 & 15 & 20 & 0.05 & 15 & 0.125 & 0.250 \\
\hline $\mathrm{G}^{3}{ }^{3}$ & 1.5 & 24 & 8 & 0.05 & 15 & 0.125 & 0.250 \\
\hline \multicolumn{8}{|l|}{ Gas } \\
\hline $\mathrm{G} 1^{1}$ & 0.6 & 6 & 1.5 & 0.1 & 11 & 0.125 & 0.625 \\
\hline $\mathrm{G}^{2}$ & 0.8 & 6 & 1.5 & 0.13 & 11 & 0.125 & 0.625 \\
\hline $\mathrm{G}^{3}{ }^{3}$ & 1.6 & 3 & 2 & 0.25 & 11 & 0.125 & 0.625 \\
\hline $\mathrm{G} 2^{1}$ & 0.6 & 3 & 3 & 0.05 & 13 & 0.125 & 0.375 \\
\hline $\mathrm{G}^{2}{ }^{2}$ & 0.8 & 5 & 3 & 0.1 & 13 & 0.125 & 0.375 \\
\hline $\mathrm{G} 2^{3}$ & 1.6 & 10 & 4 & 0.15 & 13 & 0.125 & 0.375 \\
\hline $\mathrm{G}^{1}{ }^{1}$ & 0.6 & 10 & 7 & 0.03 & 15 & 0.125 & 0.250 \\
\hline $\mathrm{G}^{2}{ }^{2}$ & 0.8 & 10 & 10 & 0.05 & 15 & 0.125 & 0.250 \\
\hline $\mathrm{G}^{3}{ }^{3}$ & 1.6 & 25 & 5 & 0.05 & 15 & 0.125 & 0.250 \\
\hline
\end{tabular}

* Gains are normalized against rated frequency and power

${ }^{1} A_{\text {nom }} \leq 500 \mathrm{kVA}$

${ }^{2} A_{\text {nom }} \leq 1500 \mathrm{kVA}$

${ }^{3} A_{\text {nom }}>1500 \mathrm{kVA}$

The regulator constants are referred to the isochronous case (zero droop). In the case of a droop controller the integral gain is set to zero and the proportional gain is determined according to the droop itself. In this last case, because the controller has a purely proportional formulation, in order to affect the controller dynamic and smooth the response, the actuator time constant is increased.

A sample of the obtained responses in case of a $1 \mathrm{MVA}$ diesel generator is depicted in Fig. 4 in the case of isochronous operation.

It is important to note that the tuning has been operated by simulating a $100 \%$ power load increase, according to the ISO standard definitions. The standard uses $100 \%$ in order to give a per unit indication, but it obviously does not require REGs to be able to actually react to a $100 \%$ power load increase, since it is not possible. However, the operated tuning is consistent because the proposed model is linear.

The sets of parameters reported in Table II define a library of "standard generators" models which can be used to simulate the frequency response of a given set of REGs using a limited
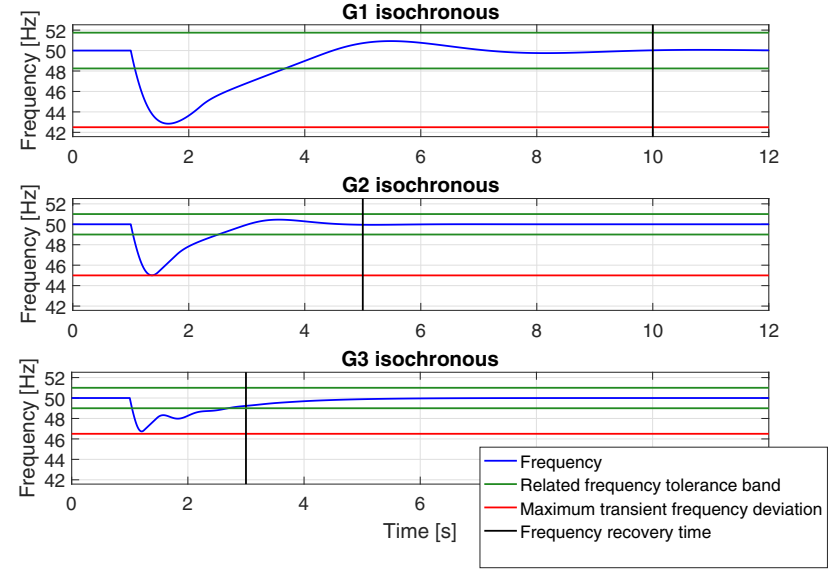

Fig. 4. 1 MVA Diesel generator frequency response obtained by using the parameter tuning summarized in Table II, according to the three different performance classes defined by [13].

amount of information. More precisely, the following data are required: number of REG units and, for each unit: nominal active power $P_{\text {nom }}$, rated apparent power $A_{\text {nom }}$, performance class, and type (Diesel or Gas). Then, once defined the parameters by Table II, the multiple unit equivalent model (4)(9) can be used to simulate the REGs set frequency response.

\section{VALidation Tests}

In order to validate the equivalent model introduced in Section II, the models of the Stamford HCI 534D and the MAK generators has been considered. The details of the models have been accurately developed and tested with the manufacturers. Table III reports the characteristics of these two generators. The relevant datasheets are in [15] and [16].

TABLE III

BENCHMARK GENERATORS MAIN PARAMETERS.

\begin{tabular}{lcc}
\hline & Stamford HCI 534D & MAK \\
\hline Fuel & Diesel & HFO \\
Voltage $[\mathrm{kV}]$ & 0.415 & 13.8 \\
$P_{\text {rated }}[\mathrm{kW}]$ & 320 & 5530 \\
$A_{\text {nom }}[\mathrm{kVA}]$ & 450 & 6912 \\
$P_{\text {min }}[\mathrm{kW}]$ & 60 & 1100 \\
Rated Mechanical Speed [rpm] & 1500 & 750 \\
Inertia Time Constant [s]* & 0.345 & 1.86 \\
\hline *Rated to $A_{\text {nom. }}$. & &
\end{tabular}

The models have been implemented on the DigSilent PowerFactory simulation platform [17]. Then, for each generator, an equivalent single unit model with the form in Fig. 1 has been identified by tuning the relevant parameters. The tuning procedure has been carried out considering a set of different tests, with positive and negative load steps. The values of the tuned parameters are summarized in the following Table IV.

Such values have been defined in order to allow the equivalent models to fit as much as possible the frequency responses obtained by the validated models, with a particular focus to the capability of reproducing the maximum transient frequency 
TABLE IV

TUNED VALUES OF THE EQUIVALENT SINGLE UNIT MODEL PARAMETERS.

\begin{tabular}{rccccccc}
\hline & $H$ & $K_{I}$ & $K_{p}$ & $\tau_{1}[\mathrm{~s}]$ & $\zeta$ & \multicolumn{2}{c}{$\tau_{2}[\mathrm{~s}]$} \\
& {$[\mathrm{s}]$} & {$[p . u]$.} & {$[p . u]$.} & & & isoch & droop \\
\hline Stamford & 0.345 & 45 & 6 & 0.01 & 15 & 0.125 & 0.250 \\
MAK & 1.86 & 3.30 & 3 & 0.15 & 7 & 0.125 & 0.250 \\
\hline
\end{tabular}

deviation (MTFD) and the frequency recovery time (FRT), as defined by ISO standard 8528-2 [13] (see Section III). According to the specifics reported in Table I, the Stamford HCI 534D results to belong to the performance class DieselG2, whereas the MAK shows performances compliant with the class Diesel-G1. Therefore, the FRT has been defined with respect to the related frequency tolerance band $\alpha_{f}=2 \%$ in the Stamford HCI 534D case, and to $\alpha_{f}=3.5 \%$ in the MAK case.

Figure 5 depicts the results of a set of the simulations carried out during the tuning procedure. It compares the frequency responses to different load steps, obtained with the detailed and equivalent models for the Stamford HCI 534D. The figures also depict the frequency bounds relative to the performance class Diesel-G2, as defined in Table I. Note that both the frequency tolerance band (dashed green lines) and the MTFD (solid green line) have been scaled up according to the load step amounts, coherently with Table I, which defines the limit values assuming a $100 \%$ load step.

It appears clear that the single unit equivalent model generally reproduces the frequency response with errors that become significant with large load steps $(\geq 20 \%$ of the rated generator power). However, it is also evident that there are no critical discrepancies in terms of MTFD and FRT. This is confirmed by the numerical data listed in Table V, which reports the absolute values of the differences between the MTFD and FRT obtained with the validated and equivalent models, respectively. The maximum FRT error is $0.54 \mathrm{~s}$, whereas the maximum MTFD error is $0.13 \mathrm{~Hz}$. However, this last value has been obtained with a $25 \%$ load step. In all other cases, the MTFD errors are lower than $0.05 \mathrm{~Hz}$.

TABLE V

VALIDATION TESTS ERRORS (ABSOLUTE VALUES).

\begin{tabular}{lcccccc}
\hline Load Steps [\%] & +7.5 & +15 & +25 & -7.5 & -15 & -25 \\
& & & & & & \\
\hline Stamford HCI 534D & & & & & & \\
FRT Errors [s] & 0.51 & 0.10 & 0.05 & 0.54 & 0.54 & 0.14 \\
MTFD Errors [Hz] & 0.01 & 0.05 & 0.13 & 0.004 & 0.01 & 0.03 \\
& & & & & & \\
MAK & & & & & & \\
FRT Err. [s] & 1.19 & 1.16 & 1.17 & 0.06 & 0.08 & 1.26 \\
MTFD Err. [Hz] & 0.03 & 0.05 & 0.06 & 0.06 & 0.12 & 0.17 \\
\hline
\end{tabular}

Table V also reports the FRT ans MTFD errors obtained within the same set of simulation scenarios, computed using the MAK models. Figure 6 depicts the results of such a set
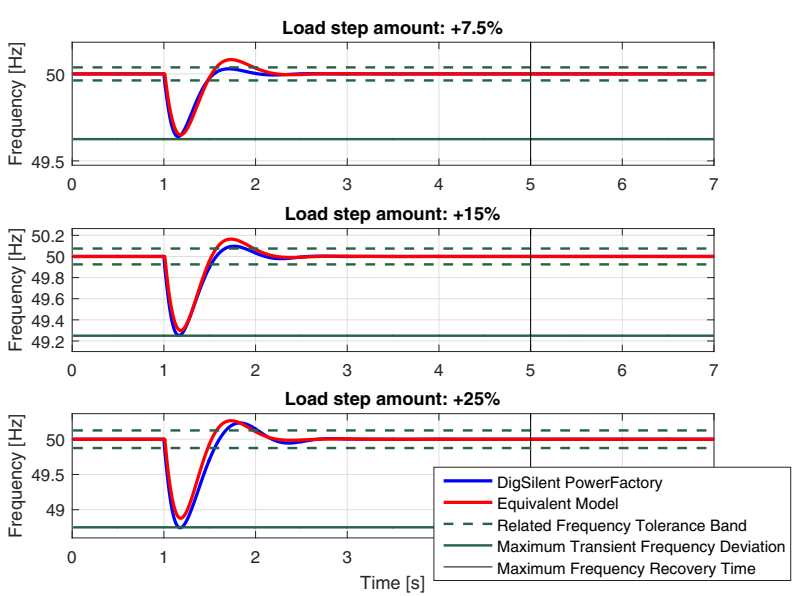

(a)
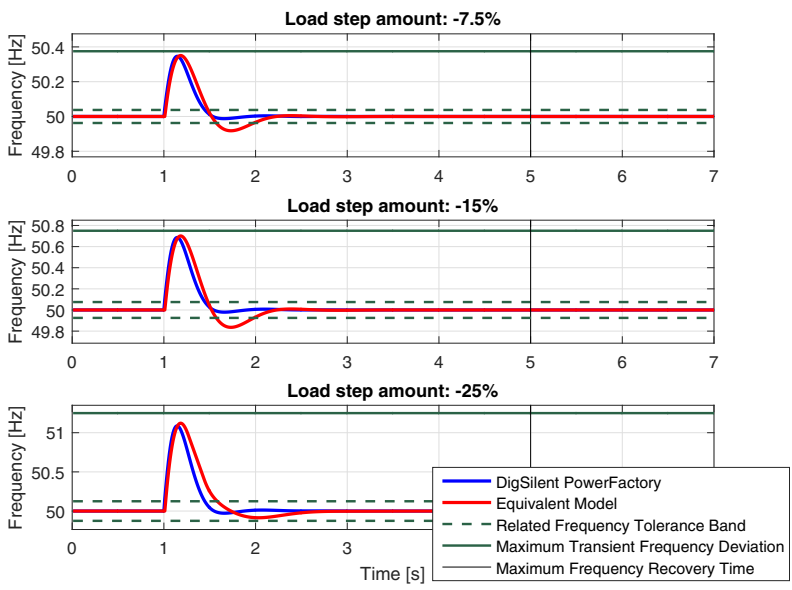

(b)

Fig. 5. Stamford HCI 534D validation results. Frequency responses to positive (a) and negative (b) load steps. The reported percentage load change amounts are defined with respect to generator nominal power. The initial generator operating point is $50 \%$. Frequency regulation is isochronous $\left(b_{p}=0\right)$. The dashed and solid green lines and the black line indicate the specifications for the ISO performance class Diesel-G2.

of simulations. In this case, the pictures report the frequency bounds relative to the performance class Diesel-G1. The results seem to be less satisfactory if compared with the Samford HCI354 ones. More precisely, the FRT errors are larger, with a maximum value equal to $1.26 \mathrm{~s}$. This is not surprising since the MAK is significantly slower than the Stamford HCI 534D. The machines sizes are indeed very different (Stamford HCI 534D is a 450 MVA generator, whereas MAK is 6912 MVA generator) and, therefore, they belong to two different performance classes, one with a maximum FRT equal to $5 \mathrm{~s}$ (Diesel-G2) and $10 \mathrm{~s}$ (Diesel-G1), respectively. Taking into account this difference, the reported results of Stamford HCI 534D and MAK can be considered as similar.

The above detailed results are two examples of the overall set of simulations carried out to validate the equivalent model. Some general conclusions can be provided. With small pertur- 

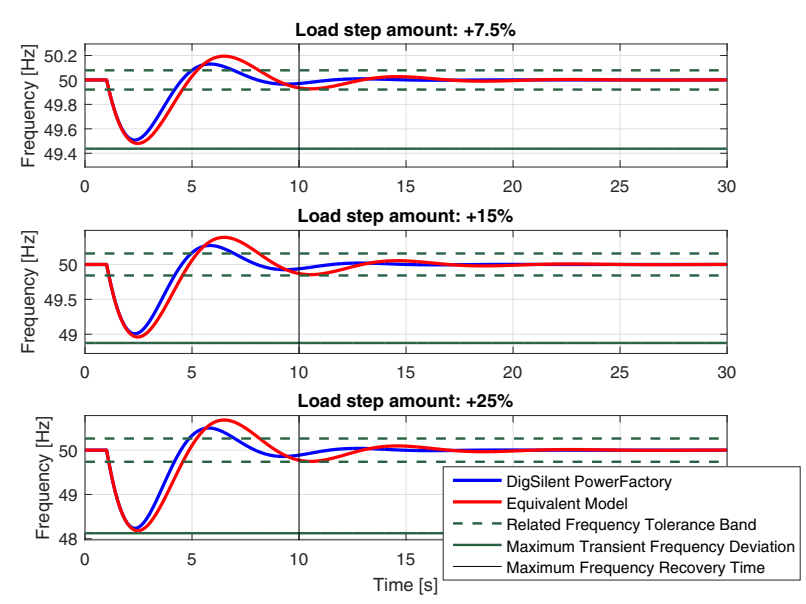

(a)
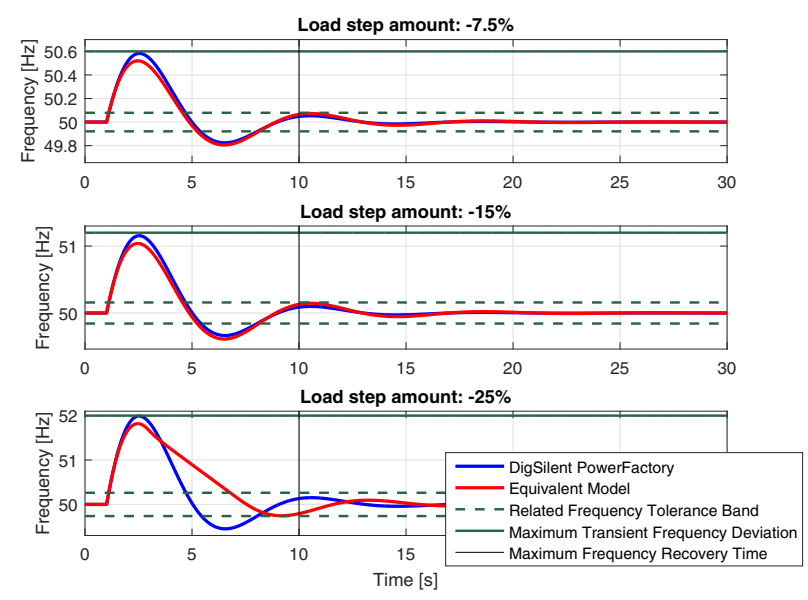

(b)

Fig. 6. Example of MAK validation results. Frequency responses to positive (a) and negative (b) load steps. The reported percentage load change amounts are defined with respect to generator nominal power. The initial generator operating point is $50 \%$. Frequency regulation is isochronous $\left(b_{p}=0\right)$. The dashed and solid green lines and the black line indicate the specifications for the ISO performance class Diesel-G1.

bations ( $\leq 20 \%$ of the rated generator power) the equivalent models are able to reproduce the generator responses with negligible errors and they result to be adequate to give a measure of the MTFD, with absolute errors lower than $0.12 \mathrm{~Hz}$ (or, more generally, $0.2 \%$ of the nominal frequency) both for Stamford HCI 534D and MAK. The FRT also is well represented, with absolute errors lower than 0.6 s for the Stamford HCI 534D and lower than $1.3 \mathrm{~s}$ for the MAK. With larger perturbations ( $>20 \%$ of the rated generator power), errors may result more significant since the equivalent model does not correctly reproduce the generator nonlinearities. However, absolute errors on the MTFD results to be generally lower than $0.3 \mathrm{~Hz}$ (or, more generally, $0.6 \%$ of the nominal frequency), whereas discrepancies on the FRT are lower than $2 \mathrm{~s}$.

\section{Conclusions}

This work has proposed a simplified and equivalent modelling approach for representing the frequency dynamics of an isolated microgrid supplied by generators driven by internal reciprocating combustion engines. The model parameters have been tuned in order to obtain a generator response compliant with the performance classes defined in the ISO 8528-5 standard [13]. The result is a library of simple models of "standard generators" which can be used to simulate the microgrid frequency dynamics. The effective capability of the proposed modelling to reproduce the frequency response of real generators has been verified using, as benchmark, the detailed and validated models of a diesel and a HFO generator.

\section{REFERENCES}

[1] N. Hatziargyriou, H. Asona, R. Irvani, and C. Marnay, "Microgrids," IEEE Power Energy Mag., vol. 5, no. 4, pp. 78-94, Jul. 2007.

[2] D. Olivares, C. Canizares, and M. Kazerani, "A centralized energy management system for isolated microgrids," Smart Grid, IEEE Transactions on, vol. 5, no. 4, pp. 1864-1875, July 2014.

[3] D. Olivares, A. Mehrizi-Sani, A. Etemadi, C. Canizares, R. Iravani, M. Kazerani, A. Hajimiragha, O. Gomis-Bellmunt, M. Saeedifard, R. Palma-Behnke, G. A. Jimenez-Estevez, and N. Hatziargyriou, "Trends in microgrid control," IEEE Trans. Smart Grid, vol. 5, no. 4, pp. 19051919, Jul. 2014

[4] G. Strbac, N. Hatziargyriou, J. C. Lopes, C. Moreira, A. Dimeas, and D. Papadaskalopoulosy, "Microgrids: Enhancing the resilience of the european megagrid," IEEE Power Energy Mag., vol. 13, no. 3, pp. 3543, May 2015.

[5] F. O. Resende, J. Matevosyan, and J. V. Milanovic, "Application of dynamic equivalence techniques to derive aggregated models of active distribution network cells and microgrids," in 2013 IEEE PowerTech, Grenoble, FR, Jun. 2013.

[6] R. Best, J. Kennedy, D. Morrow, and B. Fox, "Steady-state and transient performance of biodiesel-fueled compression-ignition-based electrical generation," IEEE Trans. Sust. Energy, vol. 2, no. 1, pp. 20-27, Jan. 2011.

[7] S. Massucco, A. Pitto, and F. Silvestro, "A gas turbine model for studies on distributed generation penetration into distribution networks," IEEE Trans. Power Syst., vol. 26, no. 3, pp. 992-999, Aug 2011.

[8] L. Minchala-Avila, A. Vargas-Martinez, L. Garza-Castan, R. MoralesMenendez, Y. Zhang, and C.-O. E., "Fault-tolerant control of a master generation unit in an islanded microgrid," in 19th IFAC World Congress, Cape Town, South Africa, Aug. 2014.

[9] B. Kuang, Y. Wang, and Y. L. Tan, "An h1 controller design for diesel engine systems," in Inter. Conf. Power System Technology, Dec. 2000, pp. 61-66.

[10] S. Roy and a. G. H. O.P. Malik, "An adaptive control scheme for speed control of diesel driven power-plants," IEEE Trans. Energy Conv., vol. 6, no. 4, pp. 605-611, Dec. 1991

[11] D. Canever, G. Dudgeon, S. Massucco, J. M. Donald, and F. Silvestro, "Model validation and coordinated operation of a photovoltaic array and a diesel power plant for distributed generation," in IEEE Summer Meeting 2001, Vancouver, Canada, Dec. 2001, pp. 626-631.

[12] F. Mohamed, "Microgrid modelling and simulation," Helsinki University of Technology, Helsinki, Finland, Tech. Rep. 47, Mar. 2006.

[13] International Standard ISO 8528-2-2005. Reciprocating internal combustion engine driven alternating current generating sets - Part 5: Generating sets, ISO, 2005.

[14] F. Saccomanno, Electric Power Systems: Analysis and Control. WileyIEEE Press, 2003.

[15] http://www.raad-eng.com/techdata/stamford/hci5d.pdf, 2015, [Online; accessed 24-June-2015].

[16] http://www.cat.com/en_US/products/new/power-systems/oil-and-gas. html, 2015, [Online; accessed 06-Sep-2016].

[17] DIgSILENT PowerFactory User's Manual, DIgSILENT GmbH, version 15.0 . 